\title{
Relationship between intraocular, \\ blood, and cerebrospinal fluid \\ pressures: a theoretical approach
}

Giovanna Guidoboni¹, Fabrizia Salerni², Rodolfo Repetto ${ }^{3}$, Marcela Szopos ${ }^{4}$, Alon Harris ${ }^{5}$

${ }^{1}$ Department of Electrical Engineering and Computer Science, University of Missouri, Columbia, MO, USA; 'Department of Mathematical, Physical and Computer Science, University of Parma, Parma, Italy; ${ }^{3}$ Department of Civil, Chemical and Environmental Engineering, University of Genoa, Genoa, Italy; ${ }^{4}$ Laboratoire MAP5, UMR CNRS 8145, Université Paris Descartes, Sorbonne Paris Cité,Paris, France; ${ }^{5}$ Institute and Department of Ophthalmology, Indiana University School of Medicine, Indianapolis, IN, USA

Keywords: blood pressure, cerebrospinal fluid pressure (CSF), intraocular pressure (IOP)

\section{Background and purpose}

Intraocular pressure (IOP), arterial blood pressure $\left(\mathrm{P}_{\mathrm{A}}\right)$, and cerebrospinal fluid (CSF) pressure have been identified as major players in several ocular pathologies, including glaucoma, central vein occlusion, and papilledema, to name a few. IOP, $P_{A}$, and CSF pressures are not independent from each other. For example, aqueous humor and CSF flows, whose mechanics contribute to establish IOP and CSF pressure levels, originate from blood flow, which is driven by $\mathrm{P}_{\mathrm{A}}$. As a consequence, it is difficult to experimentally isolate IOP, $P_{A}$, and CSF pressure and to disentangle their effect in pathological conditions. Here we utilize a theoretical approach to address this issue. 


\section{Methods}

The present mathematical model is developed to simulate fluid flow in the eyes (retina, choroid, ciliary body, and lamina cribrosa) and brain connected with a simplified description of the whole-body circulation consisting in central arteries and veins. We use an electrical circuit analog that accounts for:

1. the flows of blood and aqueous humor in the eyes;

2. the flows of blood, CSF, and interstitial fluid in the brain; and

3. lamina cribrosa biomechanics influenced by tissue pressure in the optic nerve head due to CSF within the subarachnoid space (SAS), represented by an extra ventricular compartment.

The lumped parameter circuit for the brain is adapted from the work described and validated in Lakin and Stevens ${ }^{1}$; the eye model originates from the approach proposed in Guidoboni et al. ${ }^{2}$ for the study of retinal circulation and has been extended to account for the three ocular vascular beds (retina, choroid, and ciliary body) on the basis of the work of Kiel et al. ${ }^{3}$ Finally, ocular hemodynamics is coupled with the aqueous humor dynamics as in Szopos et al. ${ }^{4}$ The model takes into account the complex interaction between different biofluids in the brain and in the eye. The flow is driven by $P_{A}$, which is given as a variable input, while the venous pressure $P_{V}$ is kept constant. Intracranial pressure (ICP) is the pressure in the brain compartment and CSF production rate is imposed and kept constant. IOP results from the balance between aqueous humor production and drainage, and acts as an external pressure on ocular vascular veins that are modeled as Starling resistors (they collapse when the transmural pressure is negative). The lamina cribrosa exerts a compressive stress from the combined action between IOP, CSF pressure, and scleral tension on translaminar central retinal arteries and veins segments as in Guidoboni et $a l^{2}$ Model parameters have been calibrated on published data. The assumptions related to the coupling between the various elements is validated: the model is used to simulate changes in IOP, CSF pressure, flow, and pressure distributions across the whole system induced by changes in $\mathrm{P}_{\mathrm{A}}$. Results are compared to those reported in clinical studies. In this current version of the model, time dependence and blood flow regulation are not included.

\section{Results}

Our model predicts relationships between IOP, $\mathrm{P}_{\mathrm{A}}$, and CSF pressure that are within the same range as those reported in clinical studies. Figure 1 captures the overall trend of CSF pressure variations due to changes in blood pressure and IOP, as reported in Ren et al. ${ }^{5}$ Morevover, as show in Table 1, the model captures the trend of IOP variation due to changes in blood pressure, as reported by various authors. ${ }^{5-9}$ In addition, choroidal venous pressure in the vortex veins computed by the model 

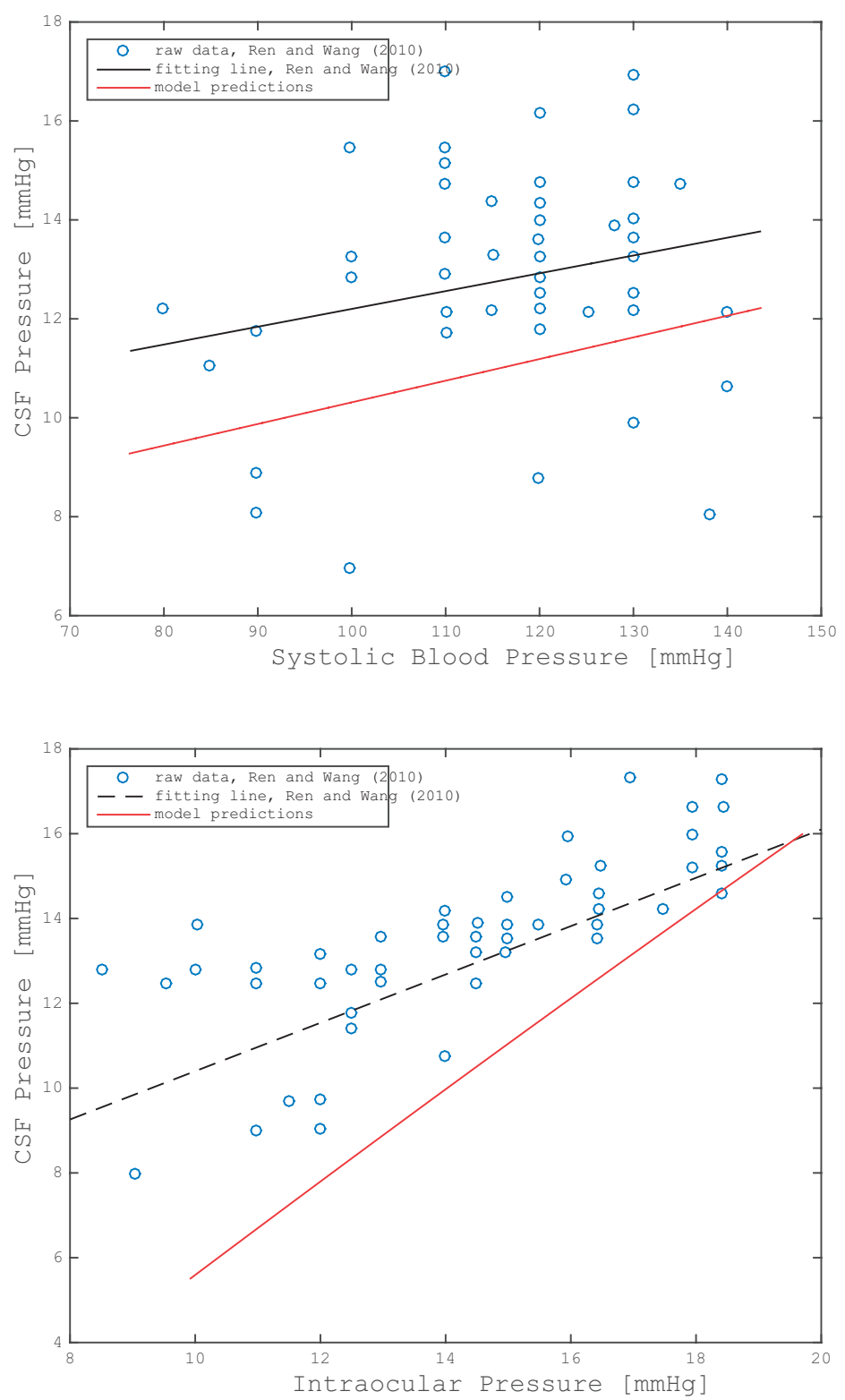

Fig. 1. (Top) Influence of systolic blood pressure on CSF pressure. (Bottom) Relationship between IOP and CSF pressure. 
Table 1. Influence of systolic and diastolic blood pressures on IOP. Comparison between population-based studies and model simulations.

\begin{tabular}{|l|l|l|}
\hline Source & $\begin{array}{l}\mathbf{m m H g} \text { increase in IOP/10 } \\
\mathbf{m m H g} \text { increase in SBP }\end{array}$ & $\begin{array}{l}\mathbf{m m H g} \text { increase in IOP/ 10 } \\
\mathbf{m m H g} \text { increase in DBP }\end{array}$ \\
\hline Dielemans et al. ${ }^{7}$ & $0.23 \pm 0.02$ & $0.24 \pm 0.04$ \\
\hline Mitchell et al. $^{8}$ & $0.28 \pm 0.05$ & $0.52 \pm 0.12$ \\
\hline Xu et al. ${ }^{9}$ & - & 0.39 \\
\hline Model Predictions & 0.42 & 0.83 \\
\hline
\end{tabular}

IOP: intraocular pressure; SBP: systolic blood pressure; DBP: diastolic blood pressure

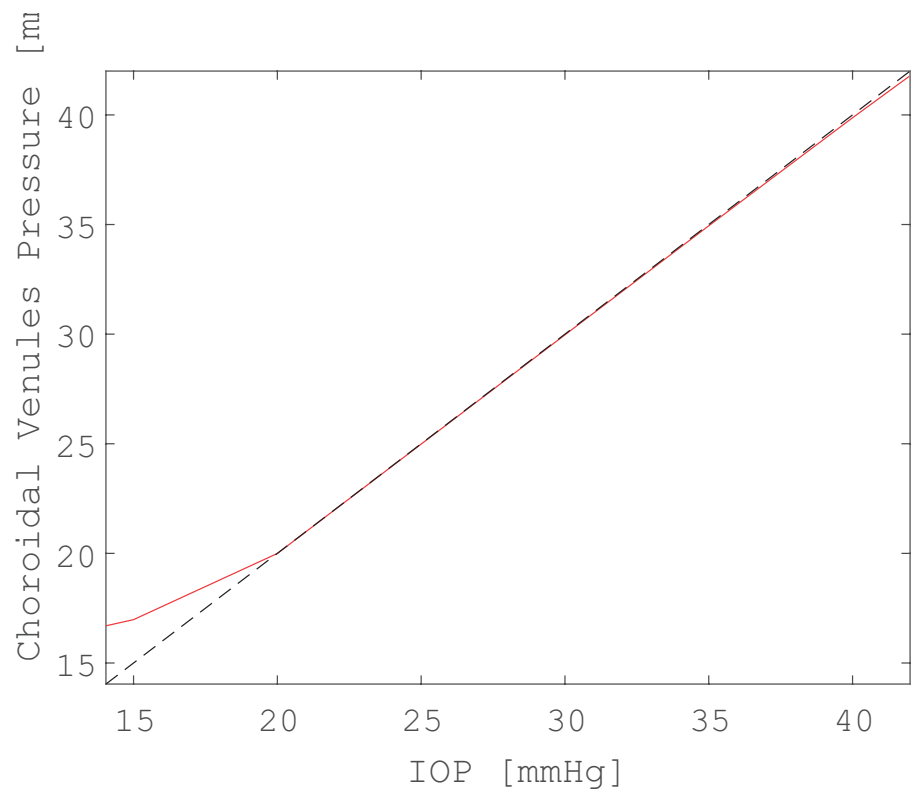

Fig. 2. Choroidal venules pressure vs IOP as predicted by the model (red curve). The black dashed line corresponds to equality between the two pressures.

from flow and pressure distribution within the body results to be approximately equal to IOP over a wide range of values (Fig. 2), confirming the findings by Bill. ${ }^{6}$ Differences between model predictions and clinical data might be due to blood flow regulation (currently not included) or particular conditions of clinical studies. 


\section{Conclusions and future perspectives}

Simulation results validate the predictive capability of the model, which provides a powerful virtual laboratory where the relationships between IOP, $\mathrm{P}_{\mathrm{A}}$, and CSF pressure can be assessed based on patient-specific conditions. Thus, our model may have an important clinical role as medicine is moving in the direction of individualized treatments for specific patients.

\section{References}

1. Lakin WD, Stevens SA. Modelling the Response of Intracranial Pressure to Microgravity Environments. Aspects of Mathematical Modelling. Springer, 2008;211-227.

2. Guidoboni G, Harris A, Cassani S, et al. Intraocular pressure, blood pressure, and retinal blood flow autoregulation: a mathematical model to clarify their relationship and clinical relevance. Invest Ophthalmol Vis Sci. 2014;55(7):4105-4118.

3. Kiel JW, Hollingsworth M, Rao R, Chen M, Reitsamer HA. Ciliary blood flow and aqueous humor production. Prog Retin Eye Res. 2011;30(1):1-17.

4. Szopos M, Cassani S, Guidoboni G, et al. Mathematical modeling of aqueous humor flow and intraocular pressure under uncertainty: towards individualized glaucoma management. Journal for Modeling in Ophthalmology. 2016;1(2):29-39.

5. Ren R, Jonas JB, Tian G, et al. Cerebrospinal fluid pressure in glaucoma: a prospective study. Ophthalmology, 2010;117(2): 259-266.

6. Bill A. The uveal venous pressure. Arch Ophthalmol. 1963;69(6):780-782.

7. Dielemans I, Vingerling JR, Algra D, Hofman A, Grobbee DE, de Jong PT. Primary open-angle glaucoma, intraocular pressure, and systemic blood pressure in the general elderly population: the Rotterdam Study. Ophthalmology. 1995;102(1):54-60.

8. Mitchell P, Lee AJ,Wang JJ, Rochtchina E. Intraocular pressure over the clinical range of blood pressure: Blue Mountains Eye Study findings. Am J Ophthalmol. 2005;140(1):131-132.

9. XuL,Wang H,Wang $\mathrm{Y}$, Jonas JB. Intraocular pressure correlated with arterial blood pressure: the Beijing Eye Study. Am. J. Ophthalmol. 2007;144(3):461-462. 